\title{
A FIXED POINT FREE NONEXPANSIVE MAP
}

\author{
DALE E. ALSPACH ${ }^{1}$
}

\begin{abstract}
In this note we give an example of a weakly compact convex subset of $L_{1}[0,1]$ that fails to have the fixed point property for nonexpansive maps. This answers a long-standing question which was recently raised again by $\mathbf{S}$. Reich [7].
\end{abstract}

1. Introduction. A (usually nonlinear) map $T$ on a subset $K$ of a Banach space $X$ is said to be nonexpansive if for every $k_{1}, k_{2}$ in $K,\left\|T k_{1}-T k_{2}\right\|<\left\|k_{1}-k_{2}\right\|$. Many authors have given conditions on the set $K$ that guarantee that a nonexpansive map $T$ on $K$ has a fixed point, e.g., [1], [2], [5], [6]. Usually $K$ is assumed to be weakly compact and convex. Of course, if $T$ is weakly continuous, then $T$ has a fixed point by the Schauder-Tychonoff fixed point theorem. For $T$ nonexpansive, (and not weakly continuous) positive results have been obtained only by placing additional requirements on $K$; however, it was unknown whether any of these additional requirements on $K$ were necessary. Our example shows that in fact some additional assumptions on $K$ are necessary.

2. The example. Let $X=L_{1}[0,1]$ and let

$$
K=\left\{f \in L_{1}[0,1]: \int_{0}^{1} f=1,0<f<2, \text { a.e. }\right\}
$$

It is easy to see that $K$ is a weakly closed, convex subset of the order interval $\{f: 0<f<2\}$, and thus $K$ is weakly compact, because order intervals in $L_{1}[0,1]$ are weakly compact. (This is a direct consequence of uniform integrability, $[3, \mathrm{p}$. 292].) Define the map $T$ from $K$ to $K$ by

$$
T f(t)= \begin{cases}2 f(2 t) \wedge 2, & 0<t<\frac{1}{2} \\ {[2 f(2 t-1)-2] \vee 0,} & \frac{1}{2}<t<1\end{cases}
$$

(We will use equality throughout with the understanding that there may be an exceptional set of measure zero.) We leave it to the reader to check that $T$ is an isometry on $K$.

Received by the editors July 15, 1980.

1980 Mathematics Subject Classification. Primary 47H10; Secondary 47H09, 54H25.

Key words and phrases. Fixed point theory.

${ }^{1}$ Research supported in part by NSF Grant MCS-7910272 
Suppose that $T$ has a fixed point $g$. We note first that $g=21_{A}$ for some set $A$ of measure one-half. Indeed,

$$
\begin{aligned}
\{t: g(t)=2\}= & \{t: \operatorname{Tg}(t)=2\} \\
= & \{t / 2: g(t)=2\}+\left\{\frac{1+t}{2}: g(t)=2\right\} \\
& +\{t / 2: 1<g(t)<2\} .
\end{aligned}
$$

(We are using + to denote disjoint union.) Because the measure of $\{t / 2: g(t)=2\}$ $+\{(1+t) / 2: g(t)=2\}$ is equal to the measure of $\{t: g(t)=2\}$, it follows that $\{t: 1<g(t)<2\}$ is of measure zero. Iteration of this argument shows that

$$
\{t: 0<g(t)<2\}=\bigcup_{n=0}^{\infty}\left\{t: 2^{-n}<g(t)<2^{-n+1}\right\}
$$

is of measure zero, as well.

Next observe that for $g=21_{A}$

$$
\left\{t: T^{n} g(t)=2\right\}=\sum_{\varepsilon_{i} \in\{0,1\}}\left\{\frac{\varepsilon_{1}}{2}+\frac{\varepsilon_{2}}{2^{2}}+\cdots+\frac{\varepsilon_{n}}{2^{n}}+\frac{t}{2^{n}}: t \in A\right\}
$$

for all $n$. We have this for $n=1$ above, and induction establishes it in general. Because $g$ is fixed, $A=\left\{t: T^{n} g(t)=2\right\}$ for all natural numbers $n$ and thus, the intersection of $\boldsymbol{A}$ with any interval with dyadic end points has measure exactly half the measure of the interval. Obviously no such measurable set exists. This contradiction shows that $T$ has no fixed point.

RemarK 1. The set $K$ has diameter two, but $\|f-1\|<1$ for all $f \in K$ and thus, $K$ cannot be the minimal weakly compact convex subset invariant under $T$. In particular, the set

$$
\bigcap_{i=1}^{\infty}\left\{f:\left\|f-\left(1+r_{i}\right)\right\|<1\right\} \cap\{f:\|f-1\|<1\} \cap K,
$$

where $r_{i}=\operatorname{sgn}[\sin 2 \pi i t$, the $i$ th Rademacher function, is invariant.

REMARK 2. It remains open whether there is a closed, bounded, convex subset of a reflexive space (hence, weakly compact) without the fixed point property for nonexpansive maps.

REMARK 3. When viewed as a transformation acting on the sets $\{(x, y): 0<y<$ $f(x)\}$. This example is essentially the baker's transformation from ergodic theory [4]. The various properties of our example can be derived from the well-known properties of that transformation.

\section{REFERENCES}

1. L. P. Belluce and W. A. Kirk, Nonexpansive mappings and fixed points in Banach spaces, Illinois J. Math. 11 (1967), 474-479.

2. F. E. Browder, Nonexpansive nonlinear operators in Banach spaces, Proc. Nat. Acad. Sci. U.S.A. 54 (1965), $1041-1044$.

3. N. Dunford and J. T. Schwartz, Linear operators: General theory, Pure and Appl. Math., vol. 7, Interscience, New York, 1958.

4. E. Hopf, Ergodentheorie, Ergebnisse der Math., Vol. 5, Springer-Verlag, Berlin, 1937.

5. L. A. Karlovitz, On nonexpansive mappings, Proc. Amer. Math. Soc. 35 (1975), 321-325.

6. E. Odell and Y. Sternfeld, $A$ fixed point theorem in $c_{0,}$ preprint.

7. S. Reich, The fixed point property for nonexpansive mappings, Amer. Math. Monthly 87 (1980), 292-294. 